\title{
Circular depolarization ratios of single water droplets and finite ice circular cylinders: a modeling study
}

\author{
M. Nicolet ${ }^{1}$, M. Schnaiter ${ }^{2}$, and O. Stetzer ${ }^{1}$ \\ ${ }^{1}$ Institute for Atmospheric and Climate Science, ETH Zurich, 8092, Zurich, Switzerland \\ ${ }^{2}$ Institute for Meteorology and Climate Research, Karlsruhe Institute of Technology (KIT), 76344 Eggenstein-Leopoldshafen, \\ Germany
}

Correspondence to: O. Stetzer (olaf.stetzer@env.ethz.ch)

Received: 8 October 2011 - Published in Atmos. Chem. Phys. Discuss.: 8 November 2011

Revised: 25 April 2012 - Accepted: 27 April 2012 - Published: 11 May 2012

\begin{abstract}
Computations of the phase matrix elements for single water droplets and ice crystals in fixed orientations are presented to determine if circular depolarization $\delta_{ \pm C}$ is more accurate than linear depolarization for phase discrimination. T-matrix simulations were performed to calculate right-handed and left-handed circular depolarization ratios $\delta_{+C}$, respectively $\delta_{-C}$ and to compare them with linear ones. Ice crystals are assumed to have a circular cylindrical shape where their surface-equivalent diameters range up to $5 \mu \mathrm{m}$. The circular depolarization ratios of ice particles were generally higher than linear depolarization and depended mostly on the particle orientation as well as their sizes. The fraction of non-detectable ice crystals $(\delta<0.05)$ was smaller considering a circular polarized light source, reaching $4.5 \%$. However, water droplets also depolarized light circularly for scattering angles smaller than $179^{\circ}$ and size parameters smaller than 6 at side- and backscattering regions. Differentiation between ice crystals and water droplets might be difficult for experiments performed at backscattering angles which deviate from $180^{\circ}$ unlike LIDAR applications. Instruments exploiting the difference in the $P_{44} / P_{11}$ ratio at a scattering angle around $115^{\circ}$ are significantly constrained in distinguishing between water and ice because small droplets with size parameters between 5 and 10 do cause very high circular depolarizations at this angle. If the absence of the liquid phase is confirmed, the use of circular depolarization in single particle detection is more sensitive and less affected by particle orientation.
\end{abstract}

\section{Introduction}

Aerosol particles in the atmosphere are essential components for cloud formation where they are known to act as cloud condensation nuclei (CCN) (Spurny, 2000). The presence of these particles influences the radiative properties of clouds and plays an important role in climate change (Lohmann and Feichter, 2005; Forster et al., 2007). The formation of ice crystals is initiated in liquid droplets by homogeneous freezing or on solid particles by heterogeneous ice nucleation mechanisms through condensation, deposition, immersion and contact freezing (Vali, 1985). However, the level of scientific understanding of those microphysical aerosol properties that determine the ice nucleation efficiency is still low (Szyrmer and Zawadzki, 1997; Cantrell and Heymsfield, 2005). One good approach to address this problem is to perform measurements of these mechanisms in the laboratory.

Continuous flow diffusion chambers (CFDC) are excellent tools to increase our understanding in these ice formation processes. The Colorado State University (CSU) instrument was the first successful device for ice nucleation studies (Rogers, 1988, 1993). The Zurich Ice Nucleation Chamber (ZINC) follows the design of the CSU chamber but uses two parallel walls instead of two concentric cylinders (Stetzer et al., 2008). It permits activation and growth of ice nuclei (IN) in an ice supersaturated environment to detect ice crystal with sizes from $1 \mu \mathrm{m}$ in diameter. As both water and liquid phases may be present during ice nucleation experiments, a detector ideally is capable of distinguishing the two phases. Depolarization of light has been suggested decades ago as a suitable method for this purpose (Fukuta and Kramer, 1968). 
The depolarization method is now widely used in remote sensing applications such as LIDARs. This technique allows discrimination between spherical and non-spherical particles. Liquid water droplets are assumed to be spherical, causing no depolarization whereas ice crystals are considered to be non-spherical and therefore imply partial depolarization of the scattered light (Liou and Schottland, 1971; Liou and Lahore, 1974). Devices using a polarized light source operating in the visible and near infrared wavelengths of the electromagnetic spectrum should be able to discriminate between liquid water and ice particles (Sassen, 1995). The depolarization technique has been used in LIDAR applications since the 1970s to detect ice crystals in clouds (Sassen, 1974, 1977; Sassen and Liou, 1979). In situ linear depolarization measurements have also been performed recently on laboratory generated ice clouds at the cloud simulation chamber AIDA (Wagner et al., 2009; Schnaiter et al., 2012). This differentiation has also been performed using a circular-polarized incident light (Bundke et al., 2008) and introduced by $\mathrm{Hu}$ et al. (2003).

Based on the same principle, an optical detector (IODE) was built to be able to distinguish between water droplets and ice crystals using linear backscattering depolarization within the ice nucleation chamber ZINC (Nicolet et al., 2010). It is based on the SIMONE detector that is used to probe aerosol and cloud particles in the large indoor chamber AIDA (Wagner et al., 2009; Schnaiter et al., 2012). Whereas the SIMONE instrument probes aerosol and cloud particle ensembles, the IODE detector aims to detect single particles as atmospheric IN concentrations are expected to be very low $\left(\sim 10 \ell^{-1}\right.$ at $\left.-25^{\circ} \mathrm{C}\right)$ (Gôtz et al., 1991) and because the detection volume of IODE is much smaller. As fixed orientations have to be taken into account, previous simulation studies of linear depolarization ratios of ice crystals showed that some orientations retrieve no depolarization. This proportion is on average between 30 and $40 \%$ and can reach up $60 \%$ to in some specific orientation cases, leading to imperfect discrimination of ice particles and water droplets (Nicolet et al., 2007).

It was suggested to use an alternative method to increase the efficiency of the phase discrimination by using circular depolarization. This technique was proposed by $\mathrm{Hu}$ et al. (2003) for LIDAR applications. This approach is based on the differences in the $P_{44}$ element of the scattering phase matrix $\mathbf{P}$ between spherical and non-spherical particles at a scattering angle $\Theta$ of $180^{\circ}$. According to Monte Carlo simulations, the phase distinction is more robust as circular depolarization is less sensitive to multiple scattering. Right-handed circularly polarized light also leads to a better discrimination of bullets, aggregates and column-like ice crystals habits than does a linearly polarized laser beam (You et al., 2006). The difference in the depolarization of scattered light by ice crystals and water droplets (defined by the ratio $P_{44} / P_{11}$ ) is also used for the Frankfurt Ice Nucleation Chamber (FINCH) where the detection is done between $\Theta=100^{\circ}$ and $130^{\circ}$
(Bundke et al., 2008). However, spheres depolarize circularly polarized light if the scattering angle is less than $\Theta=180^{\circ}$ and depolarization occurs more rapidly as we move off from perfect backscattering with increasing size parameters (Zakharova and Mishchenko, 2000).

The main objective of this paper is to compute scattering phase matrix elements $Z_{i j}$ to calculate circular depolarization ratios of single ice particles and water droplets. The influence of size, aspect ratio, and particle orientation will be investigated to see if better discrimination is still possible using circular depolarization considering the technical configuration of the IODE detector $\left(\Theta=175^{\circ}\right.$ or $\left.177^{\circ}\right)$. Calculations for randomly oriented spheroids will also be made to see if the use of circular depolarization is also possible for the SIMONE detector. Finally, the consequences of the fact that even spherical particles cause substantial depolarization for scattering angles which deviate from $180^{\circ}$ is discussed.

\section{Theory}

For a standard linear-polarization device such as the present configuration of the IODE detector (Nicolet et al., 2010), the indicent Stokes vector of the beam can be defined as $I^{\text {inc }}$ $=[1,1,0,0]$. Considering a single particle with a fixed orientation, the Stokes vector of the scattered light is given by (Mishchenko, 2000; Nicolet et al., 2007):

$$
\left[\begin{array}{c}
I^{\text {sca }} \\
Q^{\text {sca }} \\
U^{\text {sca }} \\
V^{\text {sca }}
\end{array}\right]=\frac{1}{r^{2}}\left[\begin{array}{llll}
Z_{11} & Z_{12} & Z_{13} & Z_{14} \\
Z_{21} & Z_{22} & Z_{23} & Z_{24} \\
Z_{31} & Z_{32} & Z_{33} & Z_{34} \\
Z_{41} & Z_{42} & Z_{43} & Z_{44}
\end{array}\right]\left[\begin{array}{c}
I^{\text {inc }} \\
Q^{\text {inc }} \\
0 \\
0
\end{array}\right]
$$

where $Z_{i j}$ are the elements of the phase matrix $\mathbf{Z}$. This matrix takes into account the scattering angle $\Theta$ and the Euler angles $\alpha$ and $\beta$ that describe the particle orientation (Mishchenko, 2000). The fact that the scattering matrix of spherical water droplets have the symmetry relations $Z_{11}=$ $Z_{22}$ and $Z_{12}=Z_{21}$ results in $I^{\mathrm{sca}}=Q^{\text {sca }}$, and, thus, in an non-depolarized scattering signal. For non-spherical ice crystals, in general $Z_{22} \neq Z_{11}$ and thus $I^{\text {sca }} \neq Q^{\text {sca }}$, which generates a depolarized backscattering return expressed by the linear (LIDAR) depolarization ratio $\delta_{L}=\left(Z_{11}-Z_{22}\right) /\left(Z_{11}+\right.$ $Z_{22}$ ) (Mishchenko, 2009b).

A linear polarized beam can be converted into a circular polarized one by placing a quarter-wave plate in front of the laser source. Orienting the fast-axis of the retarder in an angle $\phi=45^{\circ}$ with respect to the polarization vector of the incident linearly polarized light generates right-handed circular polarized outgoing light that can be used in light scattering applications. Hence, $I^{\text {inc }}=V^{\text {inc }}, Q^{\text {inc }}=U^{\text {inc }}=0$ and the Stokes vector becomes $[1,0,0,1]$. To analyze the circular polarized fraction of the scattered intensity, a combination of a quarterwave retarder followed by a polarizing prism is used in front of the detectors that probe the parallel $\left(I_{||}\right)$and the perpendicular $\left(I_{\perp}\right)$ linear polarization components of the scattered 

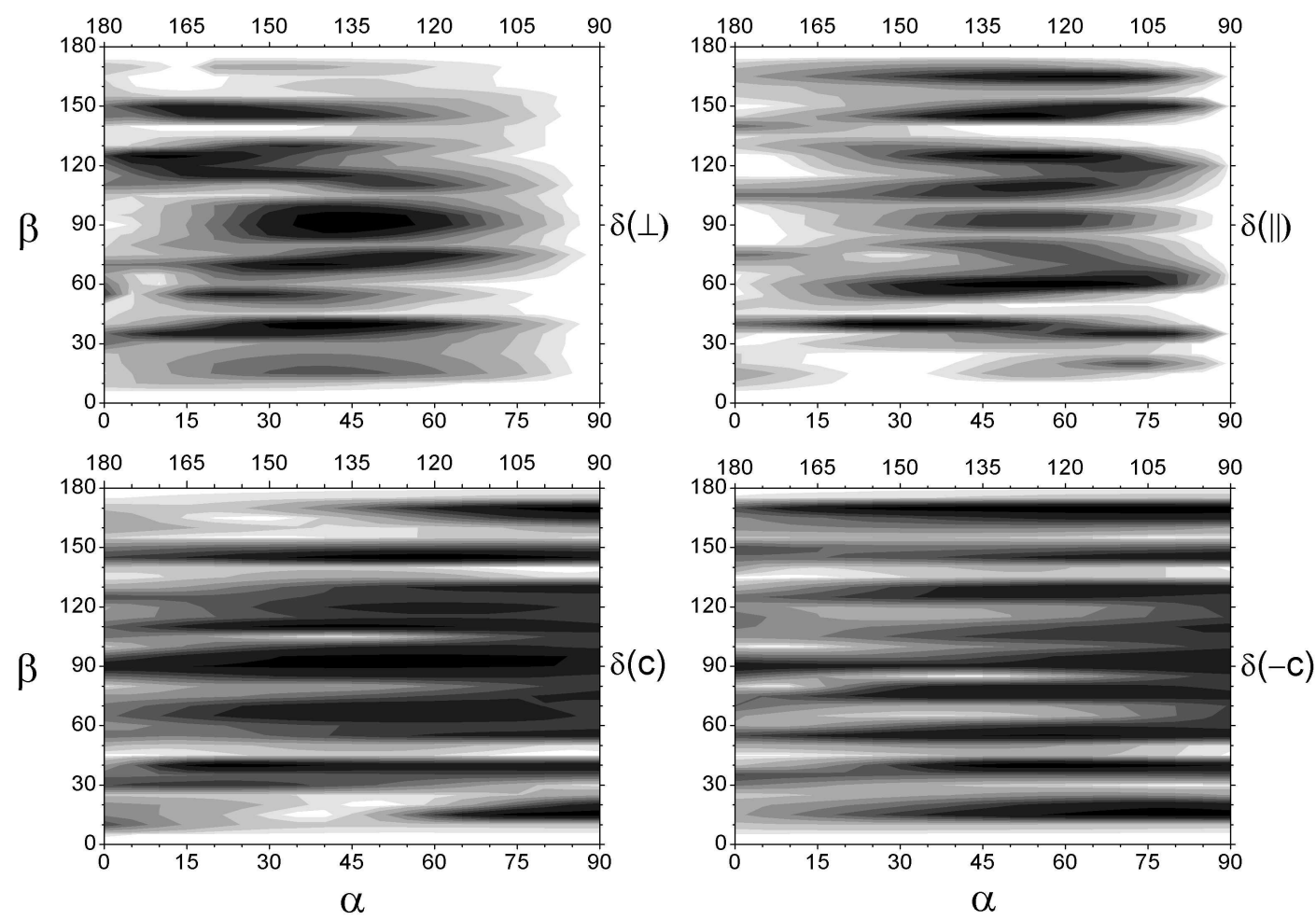

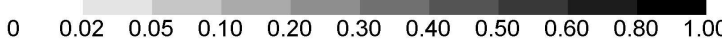

Fig. 1. Linear depolarization ratios $\delta_{\|}, \delta_{\perp}$ (upper panels) and circular depolarization ratios $\delta_{C}$ and $\delta_{-} C$ (bottom panels) for particles with $d=2 \mu \mathrm{m}$ and $\Gamma=1$ at a scattering angle of $175^{\circ}$ as a function of the particle orientation given by the Euler angles $\alpha$ and $\beta$. Note that the figure is not area representative due to the cylindrical projection.

and transmitted intensity. The generalized Stokes vector expression of the scattered light is then given by:

$\boldsymbol{I}^{\mathrm{sca}}=\frac{1}{r^{2}} \mathbf{M Z} \boldsymbol{I}^{\text {inc }}$

where $\mathbf{M}$ is the Muller matrix of the quarter-wave retarder oriented at $45^{\circ}$ and placed before the polarizing prism:

$\mathbf{M}=\left[\begin{array}{cccc}1 & 0 & 0 & 0 \\ 0 & 0 & 0 & -1 \\ 0 & 0 & 1 & 0 \\ 0 & 1 & 0 & 0\end{array}\right]$

With the above configuration the Stokes vector elements $I^{\text {sca }}$ and $Q^{\text {sca }}$, which are probed by the polarizing prism, follow as:

$I^{\mathrm{sca}}=I_{\|}^{\mathrm{sca}}+I_{\perp}^{\mathrm{sca}}=\frac{1}{r^{2}}\left(Z_{11}+Z_{14}\right)$

$Q^{\mathrm{sca}}=I_{\|}^{\mathrm{sca}}-I_{\perp}^{\mathrm{sca}}=\frac{1}{r^{2}}\left(-Z_{41}-Z_{44}\right)$

The depolarization ratio considering an incident righthanded circular polarization can be determined from Eqs. (2), (4) and (5) as:

$\delta_{+C}=\frac{I_{\perp}^{\text {sca }}}{I_{\|}^{\text {sca }}+I_{\perp}^{\text {sca }}}=\frac{Z_{11}+Z_{14}+Z_{41}+Z_{44}}{2\left(Z_{11}+Z_{14}\right)}$

Note that in contrast to the definition of $\delta$ used in LIDAR applications, we use a different definition here to restrict the upper value of $\delta$ to 1 and to be consistent with our previous study. Similarly, setting $\phi$ at $135^{\circ}$ implies that $I^{\text {inc }}=-V^{\text {inc }}$ and the Stokes vector for an incident left-handed circular polarized beam is $[1,0,0,-1]$. Consequently, the depolarization ratio in this configuration can be written as:

$\delta_{-C}=\frac{I_{\|}^{\text {sca }}}{I_{\|}^{\text {sca }}+I_{\perp}^{\text {sca }}}=\frac{Z_{11}-Z_{14}-Z_{41}+Z_{44}}{2\left(Z_{11}-Z_{14}\right)}$

At perfect backscattering $\left(\Theta=180^{\circ}\right), Z_{44} / Z_{11}=-1$ and $Z_{14}=Z_{41}=0$ which gives $\delta_{ \pm C}=0$ for spheres. For aspherical particles, $Z_{44} \neq Z_{11}$ assuming a collection of polydispersed randomly oriented ice particles (Hu et al., 2003; Liu et al., 2006), causing depolarization of circularly polarized light. The element $Z_{44}$ can be substantially different from that of spheres depending on the particle size, aspect ratio, and surface roughness (Hu et al., 2003). According to Mishchenko and Hovenier (1995), the circular depolarization 
ratio for randomly oriented particles is always greater than or equal to twice the linear depolarization ratio: $\delta_{L}<\delta_{C} \leq 2 \delta_{L}$ This fact makes the circular depolarization method attractive for LIDAR applications.

\section{Computations}

The depolarization ratio of non-spherical ice crystals in a fixed orientation was computed with the efficient T-matrix code publicly available from Mishchenko (2009a, 2000). The major difference as compared with previous studies is that we have dealt with single particles instead of a collection of randomly oriented ones. Therefore, the scattering of particles having a given specific orientation has to be considered. The source code calculates the amplitude and the phase matrices depending on the particle type (size, shape, orientation and refractive index) and the direction of both the incident and scattered light.

Ice crystals are assumed to be circular cylinders, which is an acceptable approximation at least for randomly oriented hexagonal columns (Baran et al., 2001). This approximation is acceptable also for the present single particle study, since the intention of this paper is to assess the quality of the circular depolarization measurement method for discriminating water droplets from ice crystals relative to the linear depolarization method which was investigated in the previous work by Nicolet et al. (2007).

The particle orientation can be defined by using only two Euler angles $(\alpha$ and $\beta$ ) instead of three as circular columns are axi-symmetric. Nevertheless, hexagonal particles at fixed orientations can produce complex interference effects that may not be resolved. In this simulation, particles with diameters $d$ between 0 and $5 \mu \mathrm{m}$ were simulated. This corresponds to size parameters $x=\pi d / \lambda$ between 0 and 38.6 with a IODE laser beam wavelength $\lambda$ of $407 \mathrm{~nm}$. No results can be obtained beyond this limit as the model becomes unstable and no convergence is found for larger diameters. The aspect ratio $\Gamma=d / h$ (where $h$ is the particle height) was set at 1 and $2 . \Gamma=1$ is the most realistic value, as ice crystals with $d<10 \mu \mathrm{m}$ ) tend to grow almost isometrically (Young, 1993). Concerning the orientations, the computations were performed in $5^{\circ}$-steps for each $\alpha \in\left[0,90^{\circ}\right]$ and $\beta \in\left[0,180^{\circ}\right]$ (Nicolet et al., 2007). The refractive index of ice crystals in this study is $n=1.319+2.61 \times 10^{-9} i$ (Warren, 1984).

\section{Results}

\subsection{Single finite circular cylinders}

The influence of particle orientation will first be discussed in this section. Following the previous modeling study made by Nicolet et al. (2007) for linear depolarization ratios of single ice crystals, the same representation is used for the computed circular depolarization ratios. As all orientations obtained with $\alpha \in\left[0,90^{\circ}\right]$ and $\beta \in\left[0,180^{\circ}\right]$ correspond to a quarter sphere, the regular cylindrical projection used for the contour plots exhibits overemphasized areas near the poles $\left(\beta \approx 0^{\circ}\right.$ and $180^{\circ}$ ). The following results (Fig. 1) show the linear depolarization ratios $\delta_{\|}, \delta_{\perp}$ (upper panels, taken from Nicolet et al. (2007), and circular depolarization ratios $\delta_{C}$ and $\delta_{-C}$ (bottom panels) for a particle diameter of $2 \mu \mathrm{m}$ and an aspect ratio of 1 . The scattering angle $\Theta$ is $175^{\circ}$, which refers to the older configuration of the IODE detector.

Some specific orientations of non-spherical ice crystals do not generate linear depolarization, meaning that ice particles can behave like spherical water droplets in terms of light scattering from a linearly polarized laser source. The areas where light depolarization does not occur are generally located at orientations of $\beta \approx 0^{\circ}$ and $180^{\circ}$, and $\alpha \approx 90^{\circ}$ for $d$ until $4 \mu \mathrm{m}$ and $\Gamma$ between 0.3 and 3 Nicolet et al. (2007). Circular depolarization also indicates values from 0 to 1 (= reversed rotational sense of polarization) and orientations where $\beta$ is close to $0^{\circ}$ and $180^{\circ}$ retrieve the lowest depolarization values. In contrast to linear depolarization, low circular depolarization values do not occur for $\alpha$ close to $90^{\circ}$. Moreover, the circular depolarization ratios $\delta_{C}$ and $\delta_{-C}$ are larger than the linear ones $\delta_{\|}, \delta_{\perp}$ for most of the particle orientations. Therefore, detection of ice crystals can be done more reliably as regions with low circular depolarization are less abundant than for linear depolarization. This feature is illustrated in Fig. 2 where histograms of linear and circular depolarization ratios $\delta_{\|}$and $\delta_{C}$ are given for several particle sizes and aspect ratios.

Background measurements with the SIMONE detector show depolarization ratios around 0.04 (Wagner et al., 2009; Schnaiter et al., 2012). We therefore have chosen 0.05 as a lower limit to identify an ice crystal by depolarization and distinguish it from water droplets. For linear depolarization, the fraction of ice crystals having depolarization ratios below this threshold, due to their size and orientation are within a range of $27.9 \%$ and $44.1 \%$ and get smaller with increasing particle size. In contrast, for circular depolarization, this fraction with $\delta_{C}<0.05$ remains between $4.5 \%$ and $13.8 \%$. Moreover, the distribution of circular depolarization ratios is more stable and regular, except for a diameter of $1 \mu \mathrm{m}$ where two peaks occur at [0.1-0.15] and [0.55-0.6]. In both, linear and circular cases, ice plates $(\Gamma=2)$ have better chances to be detected as ice crystals than isometrical ones considering a volume-equivalent diameter of $2 \mu \mathrm{m}$. These results confirm that the circular depolarization ratio of single ice particles is in general higher and less sensitive to the actual particle orientation than the linear depolarization ratio. However, it has a non-negligible interference with liquid droplets as discussed in the next section.

\subsection{Water droplets}

It has been shown that spheres may depolarize light if the incident laser source is circularly polarized. The ratio $Z_{44} / Z_{11}$ 

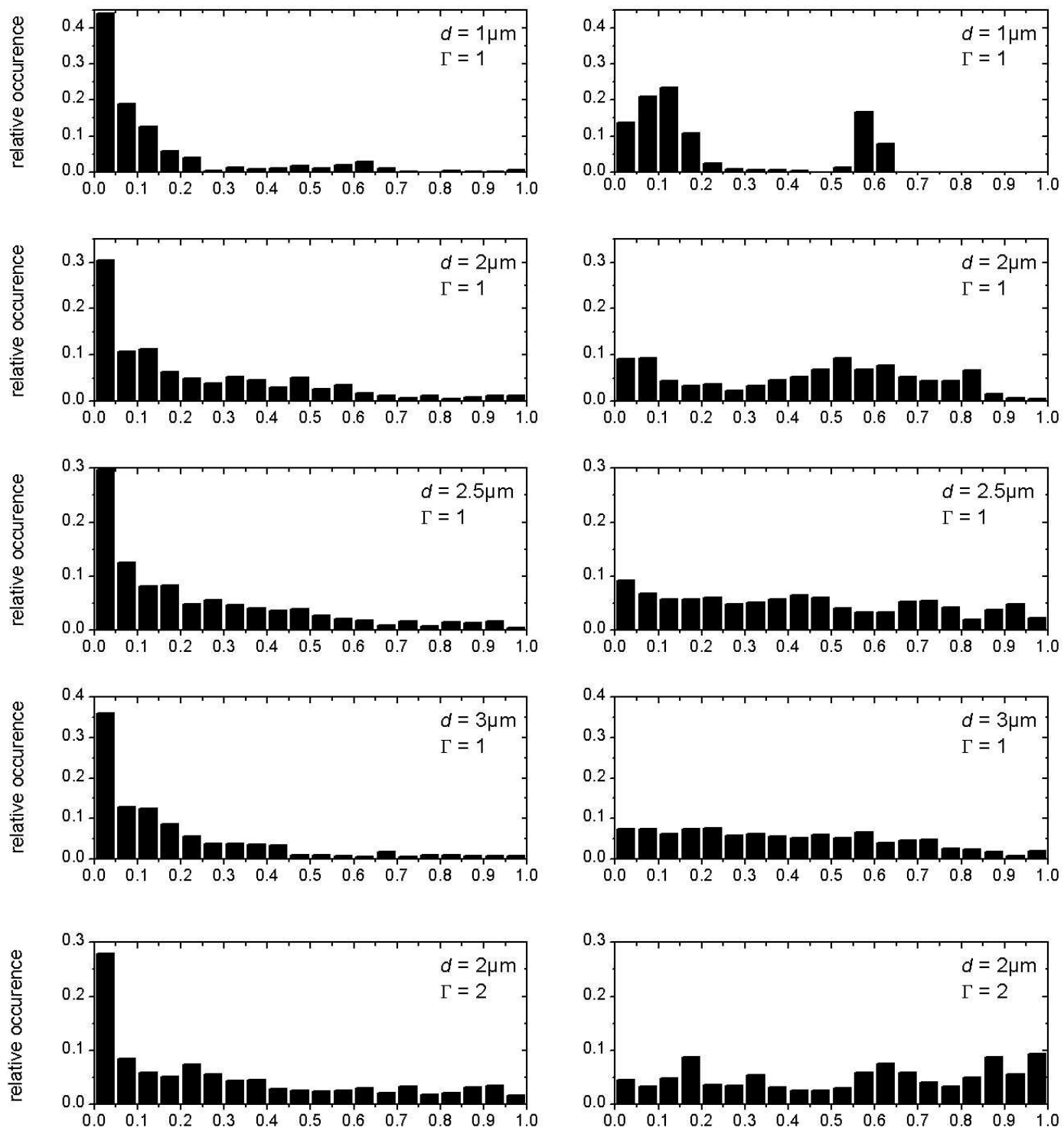

depolarization ratio $\delta(\|)$

depolarization ratio $\delta(\mathrm{c})$

Fig. 2. Occurrence of the depolarization ratio $\delta_{\|}$(left panels) and $\delta_{C}$ (right panels) considering particles diameters of $1,2,2.5$ and $3 \mu \mathrm{m}$ and aspect ratios $\Gamma$ of 1 and 2 .

differs from -1 if the scattering angle is not close to $180^{\circ}$. Depolarization caused by water droplets at specific detection geometries and sizes may be as high or even higher than depolarization ratios for ice crystals of similar sizes. Scattering matrix elements $F_{i j}$ for a hypothetical spherical particle ensemble using the refractive index of mineral dust were also investigated and the ratio $F_{44} / F_{11}$ calculated as a function of scattering angle and size parameter. Zeroand low depolarization take place at backscattering regions with small size parameters where the surface-equivalentsphere radii are given by a modified power law distribution (Mishchenko et al., 2002). Figure 3 depicts the ratio $Z_{44} / Z_{11}$ versus scattering angle and size parameter for single water droplets. Simulations performed in this case used scatteringangle steps of $0.2^{\circ}$ whereas particle diameters were considered with steps of $0.2 \mu \mathrm{m}$.

The equality $Z_{44} / Z_{11}=-1$ does not hold for nonspherical particles, but also for singles spheres. There are two negative regions at side- and backscattering angles, separated by a narrow positive branch. One major difference with Mishchenko's observations is that the side scattering region presents complex interference and resonance structures. The reason is that averaging over size is not made as single particles are considered. Therefore, there is no smoothing effect 


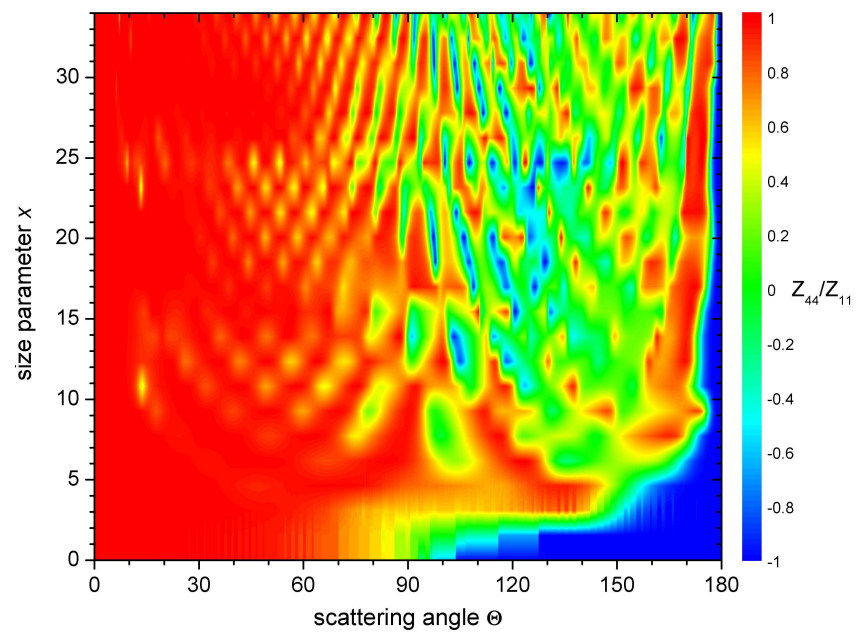

Fig. 3. $Z_{44} / Z_{11}$ ratio for spherical particles as a function of the scattering angle $\Theta$ and the size parameter $x$. The refractive index $n$ is $1.333+0 i$.

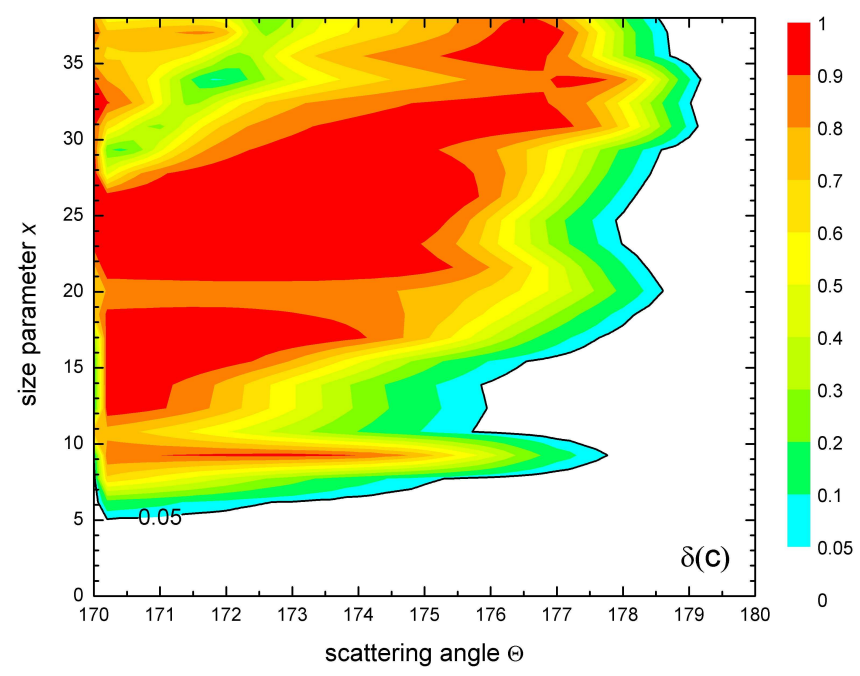

Fig. 4. Depolarization ratio $\delta_{C}$ for spherical particles as a function of the scattering angle $\Theta$ and the size parameter $x$.

on the ratio $Z_{44} / Z_{11}$ patterns (Mishchenko et al., 2002) and this parameter is strongly size dependent. The second negative region located close to $\Theta=180^{\circ}$ shows the same feature as observed by Mishchenko where water particles at perfect backscattering do not depolarize light. For forward scattering angles, the ratio is almost everywhere positive, except for isolated small regions where resonance occurs. $Z_{44} / Z_{11}$ becomes equal to unity for $\Theta=0^{\circ}$. The discontinuity steps at low size parameters at side scattering regions reflect the finite steps in $\Theta$ and $x$ in which we did our calculations.

Figure 4 illustrates the corresponding circular depolarization ratio $\delta_{C}$ zoomed at scattering angles from $170^{\circ}$ to $180^{\circ}$. As mentioned earlier in Sect. 4.1, a depolarization ratio of 0.05 is taken as a lower detection limit for ice crystals.

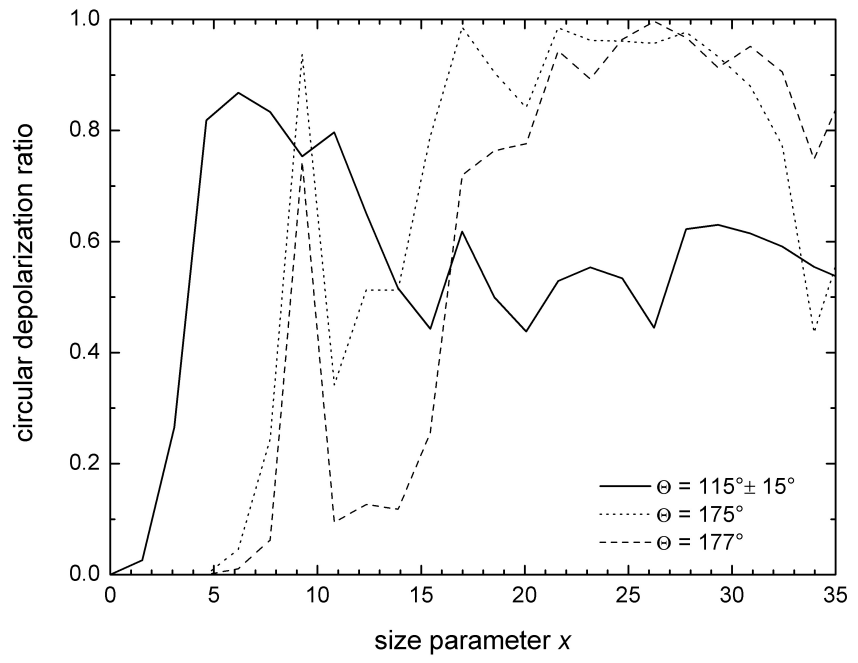

Fig. 5. Depolarization ratio $\delta_{C}$ of spheres as a function of the size parameter $x$ at $\Theta=175^{\circ}, 177^{\circ}$, and $115 \pm 15^{\circ}$.

As expected, discrimination between ice and water particles is not a problem in LIDAR applications (i.e. at $\Theta=$ $180^{\circ}$ ). This is still the case for a $1^{\circ}$ deviation from exact backscattering, except for size parameters between 30 and 35 . However, considering a scattering angle of $177^{\circ}$ for experimental use as it is the case with the IODE detector, differentiation between water droplets and ice particles can not be made for size parameters $x$ larger than $8(d \approx 1 \mu \mathrm{m})$. For the previous configuration of IODE $\left(\Theta=175^{\circ}\right)$, the size limit $(x \approx 6)$ is even lower. Consequently, the problem for distinguishing the two particle phases is the same for the SIMONE detector that is used with the AIDA chamber, as well as other laboratory devices that use circular depolarization. However, note that the SIMONE actually measures backscattering at $\Theta=178^{\circ}$ which gives a somewhat better response to spherical particles according to Fig. 4. Figure 5 summarizes the circular depolarization ratio $\delta_{C}$ at $\Theta=175^{\circ}, 177^{\circ}$ and the averaged ratio calculated between $100^{\circ}$ and $130^{\circ}$ which is used for the FINCH chamber detector (Bundke et al., 2008).

The principle for this detector is based on the difference in the $P_{44} / P_{11}$ ratio. The detection of this ratio at sidescattering angles around $115^{\circ}$ has been suggested to be more sensitive in distinguishing between a collection of spheres and a collection of non-spherical particles each following a gamma distribution (Hu et al., 2003). Bundke et al. (2008) assume that this method is also applicable for single particle detection. However, single water droplets can cause depolarization at this angle already from $x$ larger than 2 .

\section{Discussion}

We have seen that using a right-handed circular polarized incident laser source leads to a better detection accuracy of ice crystals, as the circular depolarization ratio considering all 
orientations is significantly higher than linear depolarization. In addition to that, depolarization contour plots showed that ice crystals having an orientation of $\beta \approx 0^{\circ}$ and $180^{\circ}$, and $\alpha \approx 90^{\circ}$ could be detected, using $\delta_{C}$ and $\delta_{-C}$, considering an isometric $(\Gamma=1)$ particle of $2 \mu \mathrm{m}$ in diameter. Moreover, the non-detectable occurrence of ice crystals (depolarization ratio between 0 and 0.05 ) is much lower if circular depolarization is used and this relative occurrence can be between 3 and 7 times lower. Circular depolarization is therefore often used in LIDAR applications such as CALIPSO (Winker and Wielicki, 1999) as it is less sensitive to multiple scattering (Hu et al., 2003) and discrimination between spherical and non-spherical scatterers is possible for both single and multiple scattering.

Despite the fact that circular depolarization can be used in remote sensing experiments at a backscattering angle of $\Theta=$ $180^{\circ}$, it becomes difficult to use this parameter for in-situ measurements where discrimination between water droplets and ice particles has to be done. Due to technical considerations, these instruments work at scattering angles different from $180^{\circ}$. Spherical particles depolarize light for scattering angles smaller than approximately $179^{\circ}$ and size parameters $x$ larger than $x \approx 8$, decreasing to 0 at $\Theta=180^{\circ}$. The non-depolarizing areas are also shown by Mishchenko et al. (2002) where mineral dust spherical particles are considered. Single water droplets generate interference and resonance effects at side scattering angles, leading to small regions oscillating between low- and high depolarizations and making the size dependency very high. Another way to make the solidliquid phase discrimination is to consider the difference in the $P_{44} / P_{11}$ ratio at scattering angle around $115^{\circ}$ as suggested by $\mathrm{Hu}$ et al. (2003) and used for laboratory experiments in the FINCH chamber (Bundke et al., 2008). A discrimination between water and ice seems to be possible here if the threshold value is set carefully. However, small droplets with size parameters between 5 and 10 do cause very high circular depolarizations which significantly constrains the capabilities of this detector.

\section{Conclusions}

A common technique for the discrimination between spherical water droplets and non-spherical ice particles is the measurement of the light depolarization of a linearly polarized laser source. This has been done for remote sensing applications as well as laboratory experiments. It was shown that the use of circular depolarization is more sensitive than linear depolarization. Particularly, the detection efficiency in single particle applications, like the IODE detector, is much higher. However, it has been shown that when using a circularly polarized light source only ice crystals should be present in the detection volume, e.g. by evaporating the water droplets upstream of the detector. Otherwise, water droplets with diame- ters larger than approx. $1 \mu \mathrm{m}$ lead to an overestimation of the ice particle number concentration.

These simulation results showed that measurements performed using circular depolarization would involve detection errors as water droplets might be counted as ice crystals. This problem could be counteracted by the opposite principle where ice particles are detected as water droplets due to their low depolarization ratios $(\delta<0.05)$. However, all spherical particles significantly depolarize light for $x>8$, making this solution unfeasible. Therefore, the best solution is to stick with the older configuration with a linearly polarized laser source even though the fraction of non-detectable ice particles may reach almost $45 \%$.

Acknowledgements. The authors thank M. I. Mishchenko for providing the T-matrix code for non-spherical particles in a fixed orientation. This research was supported by the Swiss National Science Foundation under grant 200021-107663/1.

Edited by: D. Baumgardner

\section{References}

Baran, A. J., Yang, P., and Havemann, S.: Calculation of the single-scattering properties of randomly oriented hexagonal ice columns: a comparison of the T-matrix and the finite-difference time-domain methods, Appl. Optics, 40, 4376-4386, 2001.

Bundke, U., Nilius, B., Jaenicke, R., Wetter, T., Klein, H., and Bingemer, H.: The Fast Ice Nucleus chamber FINCH, Atmos. Res., 90, 180-186, 2008.

Cantrell, W. and Heymsfield, A.: Production of ice in tropospheric clouds - a review, Bull. Amer. Meteorol. Soc., 86, 795, doi:10.1175/BAMS-86-6-795, 2005.

Forster, P., Ramaswamy, V., Artaxo, P., Bernsten, T., Betts, R., Fahey, D. W., Haywood, J., Lean, J., Lowe, D. C., Myhre, G., Nganga, J., Prinn, R., Raga, G., Schulz, M., and Dorland, R. V.: Climate Change 2007: The Physical Science Basis. Contribution of Working Group I to the Fourth Assessment Report of the Intergovernmental Panel on Climate Change, Cambridge University Press, Cambridge, UK and New York, USA, 2007.

Fukuta, N. and Kramer, G. K.: A fast activation continuous ice nuclei counter, J. Rech. Atmos., 3, 169-173, 1968.

Gôtz, G., Mészáros, E., and Vali, G.: Atmospheric Particles and Nuclei, Akadémiai Kiadó, Budapest, 1991.

Hu, Y. X., Yang, P., Ling, B., Gibson, G., and Hostetler, C.: Discriminating between spherical and non-spherical scatterers with lidar using circular polarization: a theoretical study, J. Quant. Spectrosc. Radiat. Transfer., 79-80, 757-764, 2003.

Liou, K. N. and Lahore, H.: Laser sensing of cloud composition: a Backscattered depolarization technique, J. Appl. Meteorol., 13, 257-263, 1974.

Liou, K. N. and Schottland, R. M.: Multiple backscattering and depolarization from water clouds for a pulsed lidar system, J. Atmos. Sci., 28, 772-784, 1971.

Liu, L., Mishchenko, M. I., Cairns, B., Carlson, B. E., and Travis, L. D.: Modeling single-scattering properties of small cirrus parti- 
cles by use of a size-shape distribution of ice spheroids and cylinders, J. Quant. Spectrosc. Radiat. Transfer., 101, 488-497, 2006.

Lohmann, U. and Feichter, J.: Global indirect aerosol effects: a review, Atmos. Chem. Phys., 5, 715-737, doi:10.5194/acp-5-7152005, 2005.

Mishchenko, M. I.: Calculation of the amplitude matrix for a nonspherical particle in a fixed orientation, Appl. Optics, 39, 10261031, 2000.

Mishchenko, M. I.: T-matrix codes for computing electromagnetic scattering by nonspherical and aggregated particles, available at: http://www.giss.nasa.gov/staff/mmishchenko/t_matrix. html, 20 January 2009a.

Mishchenko, M. I.: Electromagnetic scattering by nonspherical particles: A tutorial review, Annual Meeting of the Association-for-Aerosol-Research, Karlsruhe, Germany, 3-4 July 2008,, J. Quant. Spectrosc. Radiat. Transfer., 110, 808-832, doi:10.1016/j.jqsrt.2008.12.005, 2009b.

Mishchenko, M. I. and Hovenier, J. W.: Depolarization of light backscattered by randomly oriented nonspherical particles, Opt. Lett., 20, 1356-1358, 1995.

Mishchenko, M. I., Travis, L. D., and Lacis, A. A.: Scattering, Absorption, and Emission of Light by Small Particles, Cambridge University Press, Cambridge, UK, 2002.

Nicolet, M., Stetzer, O., and Lohmann, U.: Depolarization ratios of singles ice particles assuming finite circular cylinders, Appl. Optics, 46, 4465-4476, 2007.

Nicolet, M., Stetzer, O., Lüönd, F., Möhler, O., and Lohmann, U.: Single ice crystal measurements during nucleation experiments with the depolarization detector IODE, Atmos. Chem. Phys., 10, 313-325, doi:10.5194/acp-10-313-2010, 2010.

Rogers, D. C.: Development of a continuous flow thermal gradient diffusion chamber for ice nucleation studies, Atmos. Res., 22, 149-181, 1988.

Rogers, D. C.: Measurements of natural ice nuclei with a continuous flow diffusion chamber, Atmos. Res., 29, 209-228, 1993.

Sassen, K.: Depolarization of laser light backscattered by artificial clouds, J. Appl. Meteorol., 13, 923-933, 1974.

Sassen, K.: Ice crystal habit discrimination with the optical backscatter depolarization technique, J. Appl. Meteorol., 16, 425-431, 1977.

Sassen, K.: Lidar cloud research, Rev. Laser Eng., 23, 148-153, 1995.
Sassen, K. and Liou, K. N.: Scattering of polarized laser light by water droplet, mixed-phase and ice crystals clouds. Parts I + II, J. Atmos. Sci., 36, 838-861, 1979.

Schnaiter, M., Büttner, S., Möhler, O., Skrotzki, J., Vragel, M., and Wagner, R.: Influence of particle size and shape on the backscattering linear depolarisation ratio of small ice crystals - Cloud chamber measurements in the context of contrail and cirrus microphysics, Atmos. Chem. Phys. Discuss., in preparation, 2012.

Spurny, K.: Atmospheric condensation nuclei P. J. Coulier 1875 and J. Aitken 1880 (historical review), Aerosol. Sci. Technol., 32, 243-248, 2000.

Stetzer, O., Baschek, B., Lüönd, F., and Lohmann, U.: The Zurich Ice Nucleation Chamber (ZINC) - A new instrument to investigate atmospheric ice formation, Aerosol. Sci. Technol., 42, 6474, 2008.

Szyrmer, W. and Zawadzki, I.: Biogenic and anthropogenic sources of ice-forming nuclei: A review, B. Am. Meteorol. Soc., 78, 209228, 1997.

Vali, G.: Atmospheric ice nucleation - A review, J. Rech. Atmos., 19, 105-115, 1985.

Wagner, R., Linke, C., Naumann, K.-H., Schnaiter, M., Vragel, M., Gangl, M., and Horvath, H.: A review of optical measurements at the aerosol and cloud chamber AIDA, Annual Meeting of the Association-for-Aerosol-Research, Karlsruhe, Germany, 3-4 July 2008, J. Quant. Spectrosc. Radiat. Transfer., 110, 930-949, doi:10.1016/j.jqsrt.2009.01.026, 2009.

Warren, S. G.: Optical constants of ice from the ultraviolet to the microwave, Appl. Optics, 23, 1206-1225, 1984.

Winker, D. and Wielicki, B.: The PICASSO-CENA mission, in: Sensors, systems, and next-generation satellites III, vol. 3870, pp. 26-36, 1999.

You, Y., Kattawar, G. W., Yang, P., Hu, Y. X., and Baum, B. A.: Sensitivity of depolarized lidar signals to cloud and aerosol particle properties, J. Quant. Spectrosc. Radiat. Transfer., 100, 470-482, 2006.

Young, K. C.: Microphysical Processes in Clouds, Oxford University Press, 1993.

Zakharova, N. T. and Mishchenko, M. I.: Scattering properties of needlelike and platelike ice spheroids with moderate size parameters, Appl. Optics, 39, 5052-5057, 2000. 